\title{
Preparation and in vitro evaluation of Tacrolimus loaded liposomal vesicles by two methods: A comparative study
}

\author{
Shammy JINDAL 1,2 (D), Rajendra AWASTHI 1 (D), Dhananjay SINGARE 3 (D), Giriraj T. KULKARNI 1* (D) \\ 1 Amity Institute of Pharmacy, Amity University Uttar Pradesh, Sector-125, Noida 201313, India. \\ 2 Laureate Institute of Pharmacy, Kathog, Jawalamukhi, Himachal Pradesh 176031, India. \\ 3 Piramil Healthcare Ltd., Ahmedabad, Gujarat, India. \\ * Corresponding Author. E-mail: gtkulkarni@gmail.com (G.T.K.); Tel. +91-989-716 6866.
}

Received: 09 September 2020 / Revised: 07 December 2020 / Accepted: 19 December 2020

\begin{abstract}
Psoriasis is an autoimmune disorder characterized by hyper proliferation of the epidermal layer at the typical body sites, including nails, scalps, cleft and knees. Liposomes are the best carrier for effective delivery of bioactives in the deep skin areas. In the present study, a comparative study of liposomes was carried out using thin film hydration and curvature tuned methods. Liposomes were prepared using cholesterol, soy lecithin and Span 80. The liposomes were evaluated for physical characterization, morphology, surface charge and release properties. Liposomes prepared by the thin film hydration method (formulation TACTL2) had better properties compared to liposomes prepared by the curvature tuned method (formulation TACCT2) in terms of entrapment efficiency $(78.5 \pm 1.8 \%$ vs $53.4 \pm 5.6 \%)$, particle size $(411.8 \mathrm{~nm}$ vs $630 \mathrm{~nm})$, surface morphology(round and dispersed particles vs aggregates and lumps), and release profile $(70.1 \%$ vs $32.87 \%$ in $12 \mathrm{~h})$. Based on the results it is concluded that the thin film hydration method is better than a curvature tuned method for the preparation of liposomes.
\end{abstract}

KEYWORDS: Curvature tuned method; film hydration method; liposomes; particle size; psoriasis.

\section{INTRODUCTION}

Psoriasis is an autoimmune disease caused by the hyper proliferation of the epidermal layer of typical body sites such as nails, scalps, cleft and knees [1-2]. This is the major inflammatory skin disorder recognized by the presence of red or white patches or scales on the skin, which are very painful [3]. The interaction between genetic, and environmental factors are involved in the pathogenesis of psoriasis. However, it is difficult to explore that how the environmental factors break the body balance and affect disease onset and its development [4]. This disease affects the mental and physical quality of the life of the person. The disease is mostly associated with disorders like diabetes mellitus, hypertension, and gastrointestinal diseases, including inflammatory bowel disease, hepatic disease, infection, and mood disorders [5].

For the treatment of skin conditions various nanocarriers have been developed due to their local applications. During the past few years significant attention has been focused on the development of nanocarrier based drug delivery systems [6-10]. The development of unique and targeted therapies of existing drugs is more successful and less risky. These encapsulated systems require lower dose than the existing delivery systems [11]. Liposomal vesicles have shown promising results in topical delivery of bioactives for the effective management of various skin disorders.

Liposomes are the bilayer phospholipids molecules prepared using various fatty acids along with cholesterol and various solvents in the aqueous media. Their size range is varied from $50 \mathrm{~nm}$ to several micrometers. Liposomes can entrap both hydrophilic and lipophilic drugs, which make them extraordinary delivery system to deliver the drugs across the skin or dermal layer of the skin [12]. These are effective carrier to deliver hydrophilic and lipophilic drugs into the skin. Liposomes are prepared by several methods, namely, film hydration, ethanol injection, extrusion, bubble, french press, curvature tuned, and reverse phase evaporation. An organic solvent or detergent is used to dissolve phospholipids. Alternatively, high shear homogenization can be carried out to hydrate the phospholipids and generate liposomes. In addition to phospholipids, certain surfactants of the polyoxyethylene monoalkyl ether in combination with cholesterol also used to synthesize stable vesicles [13-17].Stability issues, irreproducibility, sterilization issues, low drug entrapment efficiency, difficult to control particle size, scale up, and rapid clearance of

How to cite this article: Jindal S, Awasthi R, Singare D, Kulkarni GT. Preparation and in vitro evaluation of Tacrolimus loaded liposomal vesicles by two methods: A comparativestudy. J ResPharm. 2021; 25(1): 34-41. 
vesicles due to non-specific uptake by the cells of reticuloendothelial system are the major limitations of liposome-based drug delivery vesicles [18].

Thin film hydration method is the simplest ways to prepare liposomes. In this method, a superimposed lipid film bilayer forms due to the evaporation of organic solvent or co-solvent in a rotary film evaporator. The lipid film is hydrated with an aqueous solution to form liposomes. Major parameters that affect the vesicle property are angle of evaporation, mass per batch, flask rotation speed and the process of hydration [19].However, thin film hydration method has limited applications for commercial scale production of liposomes due to the limited scale up capacity, and the presence of traces of organic solvent in final product [20].The $\mathrm{pH}$ jumping method, known as a curvature tuned method, is based on the phenomenon of spontaneous vesiculation and curvature theory of lipid bilayers to formulate liposomes. This method is relatively fast and solvent-free method for the preparation of stable unilamellar liposomes. However, this method also has certain limitations such as irreproducibility due to uncontrollable manufacturing conditions, low encapsulation efficiency, and potential damage of encapsulated material due to the high energy input [21]. This method is based on the rapid change in $\mathrm{pH}$, which breaks down multilamellar vesicles into unilamellar vesicles.

The aim of the present study is to compare and is to explore the thin film method and curvature tuned method for the preparation of liposomes for the topical delivery of Tacrolimus. To achieve this objective, Tacrolimus loaded liposomes were prepared using both the selected methods. The effect of formulation methods was examined in terms of particle size, polydispersity index, zeta potential, entrapment efficiency, morphological characterization, and in vitro drug release profile of Tacrolimus from the prepared liposomes.

\section{RESULTS AND DISCUSSION}

\subsection{Particle size, polydispersity index and zeta potential}

The prepared liposomes were characterized in terms of particle size, polydispersity index (PDI) and zeta potential (Table 1). For the transport of drugs to the deeper skin layer, the vesicle size should be in the range of $810 \mathrm{~nm}$ to $120 \mathrm{~nm}$. However, the penetration in deep layers of tissues increases with the decrease in vesicle size [22]. It has been reported that the temperature, duration of heating, and $\mathrm{pH}$ are major driving forces affecting particle size and their morphological properties [18].In the present study, the processing temperature for thin layer film method and curvature tuned method were $55^{\circ} \mathrm{C}$ and $60^{\circ} \mathrm{C}$, respectively. The $\mathrm{pH}$ was maintained at 7.4, and jumped from 7.4 to 11.0 in thin layer film method, and curvature tuned method, respectively. The results indicated that the vesicle size ranged from 411 to $890 \mathrm{~nm}$. The vesicle size was $411.8 \mathrm{~nm}$ and $630 \mathrm{~nm}$, respectively, for the liposomes prepared by thin layer film method (formulation TACTL2) and curvature tuned method (formulation TACCT2). The results suggested that the thin layer film method is better that the curvature tuned method in terms of vesicle size.

Table 1.Results of different physical characterization parameters of liposomal preparations.

\begin{tabular}{lcccc}
\hline $\begin{array}{l}\text { Formulation } \\
\text { code }\end{array}$ & $\begin{array}{c}\text { Weight ratio } \\
\text { (SL: CH: SP80) }\end{array}$ & $\begin{array}{c}\text { Particle size } \\
\mathbf{( n m )}\end{array}$ & $\begin{array}{c}\text { Polydispersity } \\
\text { index (PDI) }\end{array}$ & $\begin{array}{c}\text { Entrapment } \\
\text { efficiency (\%) }\end{array}$ \\
\hline TACTL1 & $7: 3: 1$ & $634.8 \pm 9.8$ & 0.402 & $69.1 \pm 0.7$ \\
TACTL2 & $8: 2: 1$ & $411.8 \pm 11.3$ & 0.327 & $78.5 \pm 1.8$ \\
TACTL3 & $9: 1: 1$ & $675.1 \pm 2.6$ & 0.385 & $59.4 \pm 0.8$ \\
TACCT1 & $7: 3: 1$ & $745.2 \pm 0.7$ & 0.402 & $49.5 \pm 0.9$ \\
TACCT2 & $8: 2: 1$ & $630.0 \pm 0.8$ & 0.322 & $53.4 \pm 5.6$ \\
TACCT3 & $9: 1: 1$ & $890.04 \pm 12.3$ & 0.510 & $35.4 \pm 0.3$ \\
\hline
\end{tabular}

TACTL: liposomes prepared by thin film hydration, and TACCT: liposomes prepared by curvature tuned method

PDI values for all the formulations were in the range of 0.327 to 0.510 (Table 1$)$. The results of PDI of vesicles prepared by the thin layer film method (formulation TACTL2) and vesicles prepared by the curvature tuned method (formulation TACCT2) are shown in Figure 1. Stability of the dispersing particles is

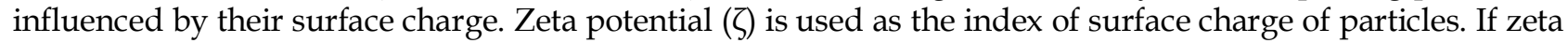
potential is high, the particles are stable due to high electrostatic repulsion between particles. On the contrary, a low zeta potential value increases the probability of forming particle aggregates. The zeta potential value of vesicles prepared by the thin layer film method (formulation TACTL2) and vesicles prepared by the curvature tuned method (formulation TACCT2) was found to be $-56.76 \mathrm{mV}$ and $-9.83 \mathrm{mV}$, 
respectively (Figure 2). The difference in the parameters such as temperature and $\mathrm{pH}$ could be the possible reason for the difference in properties of the liposomes such as particle size, polydispersity index and zeta potential in both the methods. The results suggested that the vesicles prepared by the thin layer film method were more stable than the vesicles prepared by the curvature tuned method. These two formulations were selected for further comparative study.

A

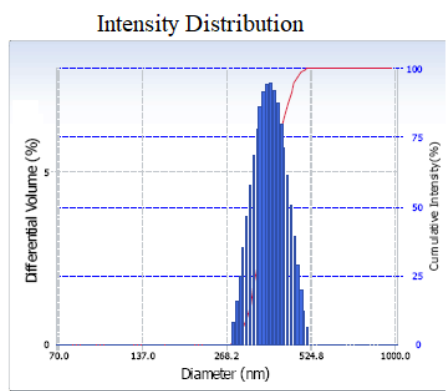

Number Distribution

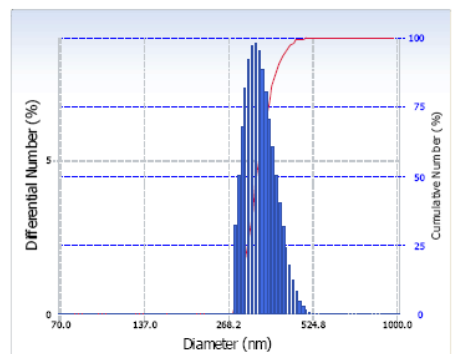

Cumulants Results

Diameter (d) : $411.8(\mathrm{~nm})$ Polydispersity Index (P.I.) : -0.327 Diffusion Const.(D): $1.195 \mathrm{e} 008\left(\mathrm{~cm}^{2} / \mathrm{sec}\right.$ $\begin{array}{lll}\text { Residual } & \text { : 4.433e003 (O.K) }\end{array}$

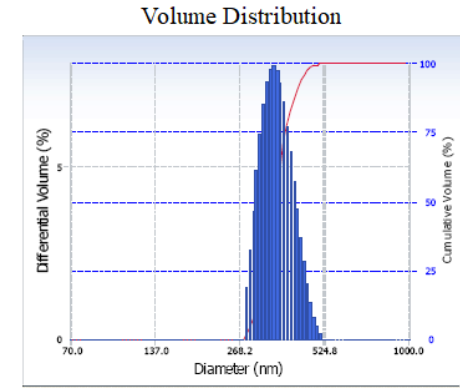

LN(G2(T)-1)vsT

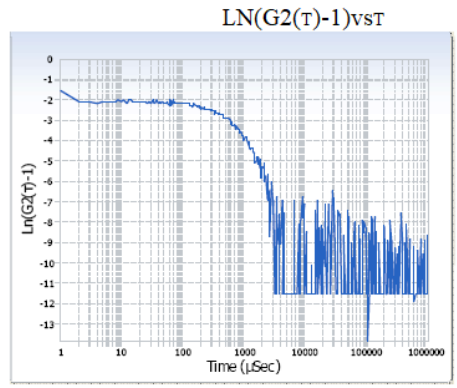

Measurment condition

Temperature $: 250\left({ }^{\circ} \mathrm{C}\right)$

Diluent Name : WATER

Refractive Index $: 1.3328$

Viscosity $: 0.8878(\mathrm{cP})$

Scattering Intensity: 9754 (cps)

B

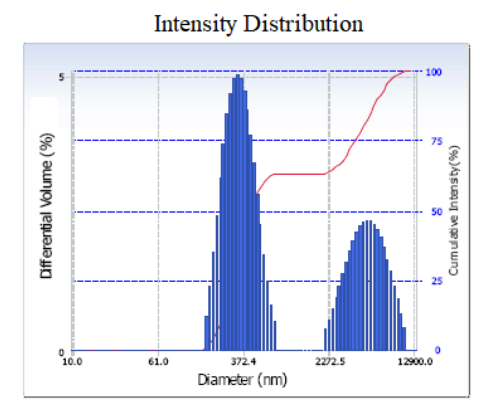

Number Distribution

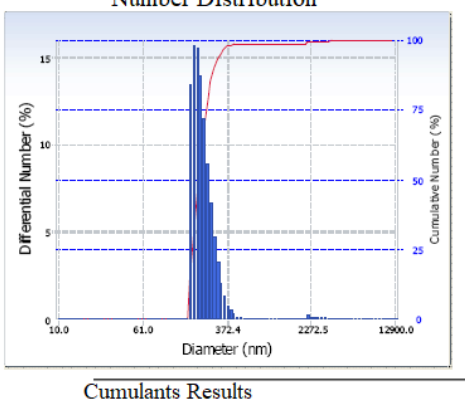

Cumulants Results

Diameter (d)

Polydispersity Index (P I ) : 0.322

Diffusion

Residual : 7.791e-003 (O.K)

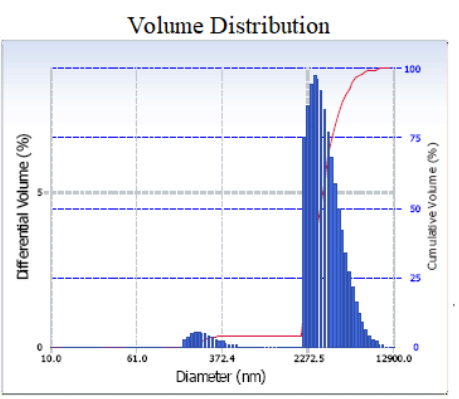

LN(G2(T)-1)vsT

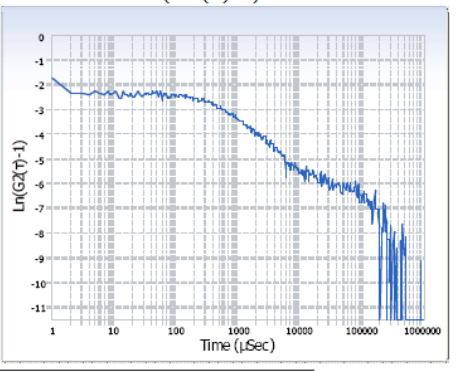

Measurment condition

Temperature $: 250 \quad\left({ }^{\circ} \mathrm{C}\right)$

Diluent Name : WATER

Refractive Index $: 1.3328$

Viscosity : $0.8878(\mathrm{cP})$

Figure 1. (A) Results of particle size distribution study of the liposomes prepared by the thin film hydration method (formulation TACTL2). (B)Results of particle size distribution study of the liposomes prepared by the curvature tuned method (formulation TACCT2). 


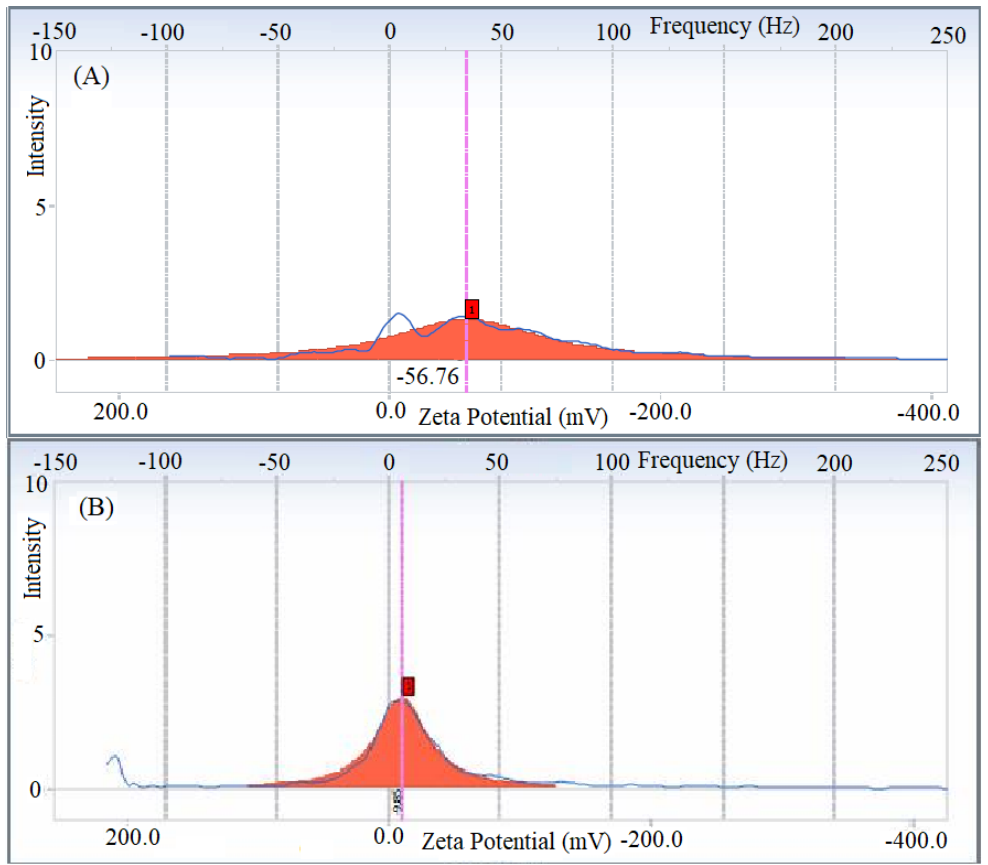

Figure 2. Results of zeta potential study of the liposomes prepared by (A) the thin film hydration method (formulation TACTL2), and (B) the curvature tuned method (formulation TACCT2).

\subsection{Entrapment efficiency}

The results of entrapment efficiency are shown in Table 1. The cholesterol concentrations significantly affected the entrapment efficiency depending on the method of preparation. Ola et al., reported increased entrapment efficiency with an increased concentration of cholesterol due to the enhanced rigidity of layer resulting in a higher stability, reduced permeability of the membrane and greater drug retention [23]. Fang et $a l$. , observed that the increased cholesterol concentration beyond a certain concentration disrupted bi-layered structure resulting in loss of drug entrapment [24].Entrapment efficiency also depends on other independent variables (hydration time, temperature, preparation method etc.) and dependent variables (particle size, morphology, particle aggregation etc.). The entrapment efficiency of vesicles prepared by the thin layer film method (formulation TACTL2) and vesicles prepared by the curvature tuned method (formulation TACCT2) was $78.5 \pm 1.8 \%$ and $53.4 \pm 5.6 \%$, respectively, despite of same concentration or ratio of formulation. Tacrolimus is a highly lipophilic compound which leads to the higher entrapment efficiency upon hydration in thin layer film method. In case of the vesicles prepared by the curvature tuned method, this behavior may be explained by the leakage of vesicle membrane due to the particle aggregation, which may facilitate the drug diffusion and thus decrease drug entrapment. This suggested that the thin layer film method is better than the curvature tuned method for the preparation of liposomes.

\subsection{Morphology}

TEM images were recorded to confirm the formation of liposomes. TEM images confirmed that the liposomes prepared by the thin layer film method (formulation TACTL2) were round and dispersed particles (Figure 3). Whereas the TEM image of liposomes prepared by the curvature tuned method (formulation TACCT2) confirmed formation of aggregates and lumps. These results are also supported from the lower zeta potential values which indicating are latively lower stability of vesicles as compared to those prepared by thin layer film method. Further, the liposomes were prepared at a higher processing temperature $\left(60^{\circ} \mathrm{C}\right)$ and $\mathrm{pH}$ jump in the curvature tuned method. The higher temperature may increase packing energy of the bilayer and thus promote aggregation of vesicles. The higher temperature and change in $\mathrm{pH}$ can also change in the shape of lipid and led to the aggregation [18]. Thus, the results suggested that the thin film hydration method is better than a curvature tuned method for the preparation of liposomal vesicles. 

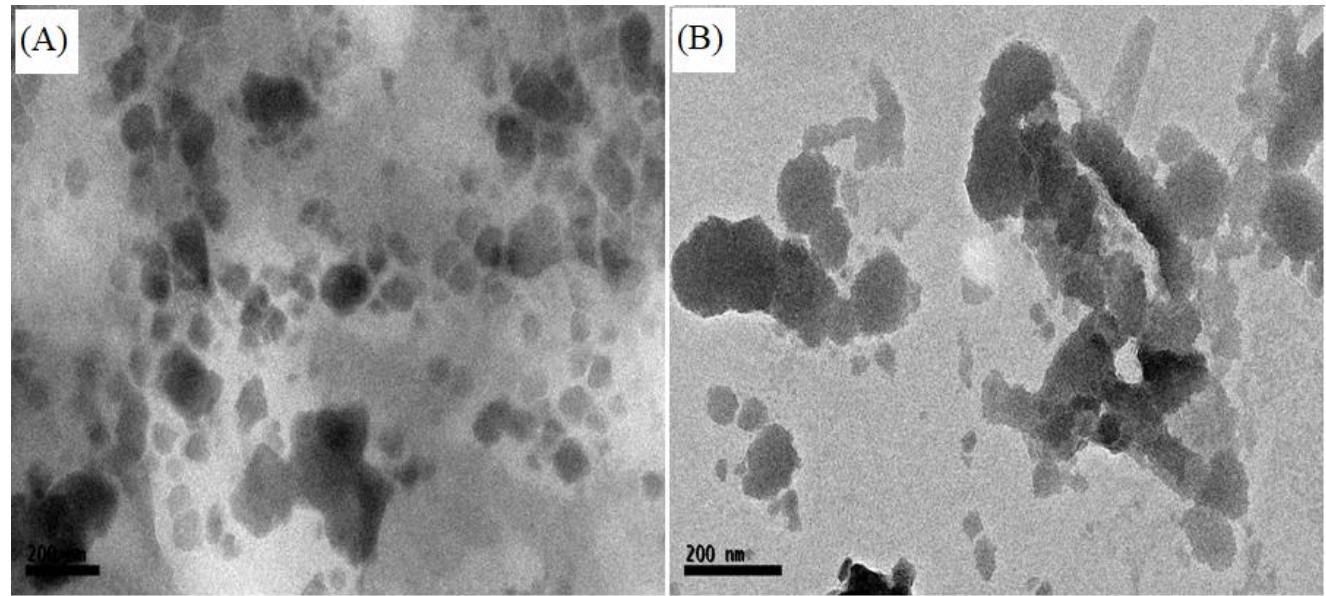

Figure 3. TEM Images of liposomes prepared by thin film hydration method (formulation TACTL2) (A), and curvature tuned method (formulation TACCT2) (B).

\subsection{In vitro release studies and release kinetics}

The benefits of topical liposomal gels are due to the maintenance of desired drug concentration for prolonged times at the site of administration [25]. In the present study, a comparative in vitro release profile of Tacrolimus from the liposomal dispersions prepared by both the methods. More sustaining release profile of Tacrolimus was observed from the liposomes prepared by the curvature tuned method. The release of Tacrolimus from vesicles prepared by the thin layer film method (formulation TACTL2) and vesicles prepared by the curvature tuned method (formulation TACCT2) was 70.1\% and 32.87\%, respectively in $12 \mathrm{~h}$ (Figure 4). The difference in release profile could be due to the change in size and morphology of liposomal vesicles prepared by both the methods. In case of the liposomes prepared by curvature tuned method, the formation of particle aggregate increases particle size and thus decreased the effective surface area. This caused a decrease in release rate from the liposomes.

To understand the kinetics governing the drug release profile, the in vitro release data was fitted into zero-order and first-order equations. The in vitro release data was fitted to the Higuchi's equation to confirm the order of release, which gives steady state drug release. To find out the drug release mechanism, and to verify the fact that whether the process of diffusion is Fickian or non-Fickian, the in vitro dissolution data was plotted according to Korsmeyer-Peppas'equation. The linearity of the plots was confirmed by the calculation of correlation coefficient $\left(\mathrm{r}^{2}\right)$. From the kinetic data of in vitro release profile, it was evident that the drug release kinetics was found to follow Higuchi's. The calculated slope values of Korsmeyer-Peppas' equitation were greater than 0.5 and less than 1, which confirmed the release through diffusion with swelling mechanism (Table 2).

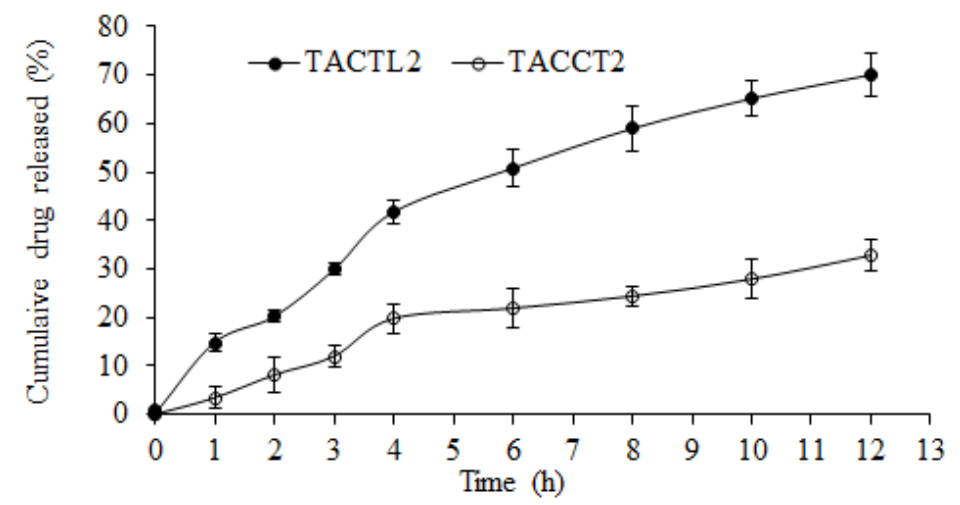

Figure 4. In vitro release profiles of Tacrolimus from the liposomes prepared by thin film hydration method (formulation TACTL2) (A), and curvature tuned method (formulation TACCT2) (B) in phosphate buffer, $\mathrm{pH} 5.5$ at $37 \pm 0.5^{\circ} \mathrm{C}$. 
Table 2. Results of kinetic equation parameters for drug release from liposomes prepared by the thin film hydration method (formulation TACTL2), and the curvature tuned method (formulation TACCT2).

\begin{tabular}{lccccc}
\hline \multirow{2}{*}{ Formulation code } & Zero order & First order & Higuchi's model & \multicolumn{2}{c}{ Peppas' model } \\
& $\mathbf{r}^{\mathbf{2}}$ & $\mathbf{r}^{\mathbf{2}}$ & $\mathbf{r}^{\mathbf{2}}$ & $\mathbf{r}^{\mathbf{2}}$ & $\mathbf{n}$ \\
\hline TACTL2 & 0.9331 & 0.9915 & 0.9808 & 0.9795 & 0.664 \\
TACCT2 & 0.9335 & 0.9892 & 0.9555 & 0.9492 & 0.870 \\
\hline
\end{tabular}

\section{CONCLUSIONS}

Liposomes are lipid bilayer membrane vesicles which have potential applications in skin targeting of drugs. Various methods have been reported for the preparation of liposomes. Each method has its own merits and demerits. In the present study, we carried out a comparative study of liposomes prepared by thin film hydration and curvature tuned methods. At laboratory scale, the thin film hydration method produced promising results in terms of vesicle size, entrapment efficiency and improved release profile. Thus, the study recommends this method for the preparation of liposomes for the dermal delivery of bioactives.

\section{MATERIAL AND METHODS}

\subsection{Materials}

Tacrolimus was obtained from M/s United Biotec Ltd., Baddi, India. Soya lecithin and cholestrol were purchased from Himedia, 23, Vadhani Industrial Estate, L.B.S. Marg, Mumbai - 400 086, India. Span 80 was purchased from Avra Laboratories Pvt. Ltd. Hydrabad, India. Sodium hydroxide, hydrochloric acid, disodium hydrogen phosphate (anhydrous, reagent grade) $\left(\mathrm{Na}_{2} \mathrm{HPO}_{4}\right)$, sodium dihydrogen phosphate (anhydrous, extra pure) $\left(\mathrm{NaH}_{2} \mathrm{PO}_{4}\right)$, and glycerol $(99.5 \%$, reagent grade) were purchased from Sigma-Aldrich Mumbai, India. Milli-Q water $(18.2 \mathrm{M} \Omega \mathrm{cm})$ was obtained using a Simplicity 185 Millipore-Water System to prepare buffer and liposomes.

\subsection{Methods}

\subsubsection{Thin film hydration method}

Soya lecithin (SL), cholesterol (CH), and/or Span 80 (SP80) along with Tacrolimus (TC) were accurately weighed and taken in a nitrogen flushed round-bottom flask containing $500 \mathrm{~mL}$ of solvent (chloroform: methanol, 2:1 v/v). The amount of drug $(0.1 \% \mathrm{w} / \mathrm{w})$ and a mass ratio of TC:SL (1:1.5) was kept constant while different SL:CH:SP80 (7:3:1, 8:2:1, 9:1:1, 7:3:2, a total mass of $100 \mathrm{mg}$ ) ratios were used to synthesize liposomes. The flask was rotated at $100 \mathrm{rpm}$. The organic solvent was evaporated using a rotary film evaporator under reduced pressure at $55^{\circ} \mathrm{C}$ to form a thin film of lipid. The flask was kept under vacuum in a desiccator to ensure complete removal of residual solvents. The obtained thin lipid film was hydrated using phosphate buffer saline $(\mathrm{pH} 7.4)$ in $10 \% \mathrm{v} / \mathrm{v}$ ethanolic solution at $55 \pm 2^{\circ} \mathrm{C}$. The resulting dispersion was kept in bath sonicator and vortexed for $10 \mathrm{~min}$ and $5 \mathrm{~min}$, respectively, and kept undisturbed for 2-3 $\mathrm{h}$ for complete hydration.

\subsubsection{Curvature tuned method}

A lipid mixture (as used in thin film hydration method) was hydrated in $10 \mathrm{~mL}$ of media (Milli-Q water). The temperature was maintained at $60^{\circ} \mathrm{C}$. The mixture was vortexed in a $10 \mathrm{~mL}$ falcon tube (with glass beads) for 1-3 min and $6 \mathrm{~mL}$ of the buffer solution ( $\mathrm{pH} 7.4)(3 \% \mathrm{v} / \mathrm{v}$ glycerol) was added. Glycerol interacts with the polar head group of lipids and produce antioxidative property. This allows long-term storage of the liposomal formulations [26]. The mixture was stirred for $15 \mathrm{~min}$ at constant temperature. The $\mathrm{pH}$ was subsequently increased to a maximum ( $\mathrm{pH} 11)$ using $\mathrm{NaOH}$ and adjusted to $\mathrm{pH} 7.4$ using $\mathrm{HCl}$ for a fixed time ( $\mathrm{pH}$ jumping time, $\Delta \mathrm{jt}$ ). The resulting mixture was stirred to achieve equilibration ( $\Delta$ eq). Finally, the stirring and heating was stopped, and the mixture was cooled to room temperature. The resultant mixture was centrifuged at $1500 \mathrm{rpm}$ for $15 \mathrm{~min}$ to collect liposomes and stored at $4{ }^{\circ} \mathrm{C}$.

\subsection{Characterization of liposomes}

\subsubsection{Determination of particle size, polydispersity index andzeta potential}

The mean particle size and poly dispersity index (PDI) were recorded for the liposomes prepared by both methods. Particle size and size distribution of Tacrolimus liposomes were determined by dynamic light 
scattering technique. Before analysis, the samples were diluted with millipore water at 1:10 dilution. The zeta potential values were determined using Delsa Nano 4C model of Beckman Coulter, Inc., USA.

\subsubsection{Determination of entrapment efficiency}

Liposomal formulations were centrifuged at 15,000 rpm for $15 \mathrm{~min}$ to obtain pellet. To disrupt the liposomes, the samples were treated with $0.01 \%$ Triton X-100 [27]. Methanol $(0.5 \mathrm{~mL})$ was added to solubilize the loaded drug. The samples were centrifuged at 10,000 rpm for $7 \mathrm{~min}$. The concentration of the encapsulated drug was determined using HPLC method with Phenomenex C18 column (75 x 3 mm I.D., 2.6 $\mu \mathrm{m})$. the analysis was performed using mixture of water with $0.1 \%$ Triflouroacetic (TFA) acid and acetonitrile with $0.1 \% \mathrm{v} / \mathrm{v}$ TFA with a flow rate of $1 \mathrm{~mL} / \mathrm{min}$ over $7 \mathrm{~min}$ and detection at $205 \mathrm{~nm}$. The instrument was shimadzu prominence LC 20 AD with lab solution software used to estimate the results. (Instrument details). The entrapment efficiency was calculated using below formula [Eq. 1]:

Entrapment efficiency $(\%)=\frac{\text { Amount of drug entrapped }}{\text { Total amount of drug taken }} \times 100$

[Eq. 1]

\subsubsection{Morphological characterization}

Morphological characterization of the prepared liposomes was carried out using transmission electron microscopy (TEM) (JEM2100, JEOL, Japan). The liposomal dispersion was placed on a Formvar ${ }^{\circledR}$ carbon coated copper grid and allowed to dry. The samples were stained with uranyl acetate and dried. The images of dried samples were recorded using Digital Micrograph ${ }^{\circledR}$ software (Gatan, Inc., USA).

\subsubsection{In vitro release studies}

In vitro release study of Tacrolimus from liposomes was carried out using Franz diffusion cell [28]. Briefly, $1 \mathrm{~g}$ of formulation were taken into the donor compartment. The donor compartment was placed in $15.0 \mathrm{~mL}$ of release media (phosphate buffer, $\mathrm{pH} 5.5$ ) and stirred at $100 \mathrm{rpm}$. The temperature was maintained at $32 \pm 0.5^{\circ} \mathrm{C}$ [25]. Dialyzing medium $(1 \mathrm{~mL})$ was withdrawn at predetermined time intervals $(0,1,2,3,4,6,8$, 10 and $12 \mathrm{~h}$ ) followed by immediate replacement with fresh media to maintain sink condition and the drug was analyzed by HPLC.

To estimate the precise drug release kinetics from the prepared liposomes the in vitro drug release data was analyzed for release kinetics models viz. zero order (cumulative amount of drug released against time) and first order (log cumulative percentage of drug remaining against time) models. The drug release mechanism was confirmed using Higuchi's (cumulative percentage drug release against the square root of time) and Korsemeyer-Peppas' (log cumulative percentage of drug release against time) equations [29].

Author contributions: Concept -G.T.K.; Design -R.A., D.S., G.T.K.; Supervision -G.T.K., R.A., D.S.; Resources - S.J.; Materials - R.A.; Data Collection and/or Processing - S.J., R.A.; Analysis and/or Interpretation - D.S., G.T.K.; Literature Search - S.J., R.A.; Writing - S.J., R.A., G.T.K.; Critical Reviews - S.J., R.A., D.S., G.T.K.

Conflict of interest statement: The authors declared no conflict of interest in the manuscript.

\section{REFERENCES}

[1] Ramanunny AK, Wadhwa S, Singh SK, Sharma DS, Khursheed R, Awasthi A. Treatment strategies against psoriasis: principle, perspectives and practices. Curr Drug Deliv. 2020; 17(1):52-73. [CrossRef]

[2] Jindal S, Awasthi R, Singhare D, Kulkarni GT. Topical delivery of Tacrolimus using liposome containing gel: An emerging and synergistic approach in management of psoriasis. Med Hypotheses. 2020; 142: 109838. [CrossRef]

[3] Deng Y, Chang C, Lu Q. Theinflammatoryresponse in psoriasis: a comprehensivereview. Clin Rev Allergy Immunol. 2016; 50(3):377-389. [CrossRef]

[4] Zeng J, Luo S, Huang Y, Lu Q. Critical role of environmental factors in the pathogenesis of psoriasis. J Dermatol. 2017; 44(8): 863-872. [CrossRef]

[5] Takeshita J, Grewal S, Langan SM, Mehta NN, Ogdie A, Van Voorhees AS, Gelfand JM. Psoriasis and comorbid diseases part I. Epidemiology. J Am Acad Dermatol. 2017: 76(3); 377-390. [CrossRef] 
[6] Badri W, Eddabra R, Fessi H, Elaissari A. Biodegradable polymer based nanoparticles: Dermal and transdermal drug delivery. J Colloid SciBiotechnol.2014; 3(2): 141-149. [CrossRef]

[7] Ramos Campos EV, Proença PL, Doretto-Silva L, Andrade-Oliveira V, Fraceto LF, de Araujo DR. Trends in nanoformulations for atopic dermatitis treatment. Expert Opin Drug Deliv. 2020. [CrossRef]

[8] Ingebrigtsen SG, Škalko-Basnet N, Holsæter AM. Development and optimization of a new processing approach for manufacturing topical liposomes-in-hydrogel drug formulations by dual asymmetric centrifugation. Drug Dev Ind Pharm. 2016; 42(9): 1375-1383. [CrossRef]

[9] Sala M, Diab R, Elaissari A, Fessi H. Lipid nanocarriers as skin drug delivery systems: Properties, mechanisms of skin interactions and medical applications. Int J Pharm. 2018; 535(1-2): 1-7. [CrossRef]

[10] Jada A.A special issue on inorganic colloidal particles, synthesis, surface properties and applications. J Colloid SciBiotechnol.2014; 3: 1-2. [CrossRef]

[11] Tiwari G, Tiwari R, Sriwastawa B, Bhati L, Pandey S, Pandey P, Bannerjee SK. Drug delivery systems: An updated review.Int J Pharm Investig. 2012; 2(1): 2-11. [CrossRef]

[12] Sherry M, Charcosset C, Fessi H, Greige-Gerges H. Essential oils encapsulated in liposomes: A review. J Liposome Res. 2013; 23(4): 268-275. [CrossRef]

[13] Sercombe L, Veerati T, Moheimani F, Wu SY, Sood AK, Hua S. Advances and challenges of liposome assisted drug delivery. Front Pharmacol. 2015; 1(6):286. [CrossRef]

[14] Bangham AD, Standish MM, Watkins JC. Diffusion of univalent ions across the lamellae of swollen phospholipids. J Mol Biol. 1965; 13(1):238-252. [CrossRef]

[15] Woodbury DJ, Richardson ES, Grigg AW, Welling RD, Knudson BH. Reducing liposome size with ultrasound: Bimodal size distributions. J Liposome Res. 2006; 16(1): 57-80. [CrossRef]

[16] Ong SGG, Chitneni M, Lee KS, Ming LC, Yuen KH. Evaluation of extrusion technique for nanosizing liposomes. Pharmaceutics. 2016; 8(4): 36. [CrossRef]

[17] Patil YP, Jadhav S. Novel methods for liposome preparation. Chem Phys Lipids. 2014; 177: 8-18. [CrossRef]

[18] Genc R, Ortiz M, O Sullivan CK. Curvature-tuned preparation of nanoliposomes. Langmuir. 2009; 25(21): 1260412613. [CrossRef]

[19] Sharma A, Sharma US. Liposomes in drug delivery: progress and limitations. Int J Pharm. 1997; 154(2): 123-140.

[20] Awasthi R, Kumar S, Kulkarni GT. Frontier lipid-based carrier systems for drug targeting: A laconic review on niosomes. Pharm Nanotechnol. 2014; 2(3): 116-128.

[21] Varona S, Martin A, Cocero MJ. Liposomal incorporation of lavandin essential oil by a thin-film hydration method and by particles from gas-saturated solutions. IndEngChem Res. 2011; 50(4): 2088-2097. [CrossRef]

[22] Verma DD, Verma S, Blume G, Fahr A. Particle size of liposomes influences dermal delivery of substances into skin. Int J Pharm. 2003; 258(1-2):141-151. [CrossRef]

[23] Ola H, Yahiya SA, El-Gazayerly ON. Effect of formulation design and freeze-drying on properties of fluconazole multilamellar liposomes. Saudi Pharm J. 2010; 18(4): 217-224. [CrossRef]

[24] Fang JY, Hong CT, Chiu WT, Wang YY. Effect of liposomes and niosomes on skin permeation of enoxacin. Int J Pharm. 2019; 219(1-2):61-72. [CrossRef]

[25] Dragicevic-Curic N, Winter S, Stupar M, Milic J, Krajišnik D, Gitter B, Fahr A. Temoporfin-loaded liposomal gels: Viscoelastic properties and in vitro skin penetration. Int J Pharm. 2009; 373(1-2): 77-84. [CrossRef]

[26] Lasic DD. The mechanism of vesicle formation. Biochem J. 1988; 256(1):1-11. [CrossRef]

[27] Laouini A, Jaafar-Maalej C, Limayem-Blouza I, Sfar S, Charcosset C, Fessi H. Preparation, characterization and applications of liposomes: State of the art. J Colloid Sci. 2012: 1(2): 147-168. [CrossRef]

[28] Katiyar SS, Muntimadugu E, Rafeeqi TA, Domb AJ, Khan W. Co-delivery of rapamycin-and piperine-loaded polymeric nanoparticles for breast cancer treatment. Drug Deliv. 2016; 23(7): 2608-2616. [CrossRef]

[29] Khan W, Kumar N. Drug targeting to macrophages using paromomycin-loaded albumin microspheres for treatment of visceral leishmaniasis: An in vitro evaluation. J Drug Target. 2011; 19(4): 239-250. [CrossRef] 